PROCEEDINGS OF THE

AMERICAN MATHEMATICAL SOCIETY

Volume 135, Number 3, March 2007, Pages 921-927

S 0002-9939(06)08662-X

Article electronically published on October 11, 2006

\title{
MORSE-PALAIS LEMMA FOR NONSMOOTH FUNCTIONALS ON NORMED SPACES
}

\author{
DUONG MINH DUC, TRAN VINH HUNG, AND NGUYEN TIEN KHAI
}

(Communicated by Jonathan M. Borwein)

\begin{abstract}
Using elementary differential calculus we get a version of the Morse-Palais lemma. Since we do not use powerful tools in functional analysis such as the implicit theorem or flows and deformations in Banach spaces, our result does not require the $C^{1}$-smoothness of functions nor the completeness of spaces. Therefore it is stronger than the classical one but its proof is very simple.
\end{abstract}

\section{INTRODUCTION}

In [9, Palais proved the Morse-Palais lemma for $C^{3}$ functions. This result was extended for $C^{2}$ functions by Kuiper in [6] (see also [8]). The Morse-Palais lemma gives us the similarity of the shapes of the graphs of $J(x)$ and $D^{2} J(0)(x, x)$, consequently the existence of the second derivatives is essential. Recently $\mathrm{Li}, \mathrm{Li}$, and $\mathrm{Liu}$ [7] obtained a version of the Morse-Palais lemma without the $C^{2}$-smoothness. Now we consider perturbed problems as follows: we study the multiplicity of solutions to the equation

$$
-\Delta u-\lambda u=f(x, u), \quad x \in \Omega,
$$

with the corresponding functional $J=J_{1}-J_{2}$, where $\Omega$ is a bounded smooth open domain in $\mathbb{R}^{n}, J_{1}(u)=\frac{1}{2} \int_{\Omega}\left(|\nabla u|^{2}-\lambda|u|^{2}\right) d x$ and $J_{2}(u)=\int_{\Omega} \int_{0}^{u(x)} f(x, s) d s d x$. The problem $(P)$ is the main application in [7].

The functional $J_{1}$ is smooth and we can investigate the shape of the graph of $D^{2} J_{1}(0)(x, x)$. But the perturbed part $J_{2}$ may not be $C^{1}$-smooth. We observe that $D^{2} J_{1}(0)$ is very nice: there exist a closed vector subspace $W^{+}$and a finitedimensional vector subspace $W^{-}$of the Sobolev space $W_{0}^{1,2}(\Omega)$ such that $W^{+} \oplus W^{-}$ is a direct decomposition of $W_{0}^{1,2}(\Omega)$ and $D^{2} J_{1}(0)(y, y) \leq 0 \leq D^{2} J_{1}(0)(x, x)$ for any $(x, y)$ in $W^{+} \times W^{-}$.

By this observation we try to find a special version of the Morse-Palais lemma for problems similar to $(P)$, in which we do not need any second derivatives. In the present paper we get this version, which does not require the $C^{1}$-smoothness of functions nor the completeness of spaces as follows.

Theorem 1.1. Let $(H,\|\|$.$) be a normed vector space and let J$ be a continuous and continuously directional differentiable real function on an open ball $B_{H}(0,2 \delta)$ in $H$,

Received by the editors October 6, 2005.

2000 Mathematics Subject Classification. Primary 58E05, 35J20.

Key words and phrases. Morse-Palais lemma, normed spaces, directional differentiability.

(C)2006 American Mathematical Society 921

Reverts to public domain 28 years from publication 
that is, $D J(x)(h)=\lim _{t \rightarrow 0} \frac{J(x+t h)-J(x)}{t}$ exists for any $(x, h)$ in $B_{H}(0,2 \delta) \times H$ and the map $x \mapsto D J(x)(h)$ is continuous on $B_{H}(0,2 \delta)$ for any fixed $h$ of $H$ and $D J(x)$ is a linear mapping from $H$ into $\mathbb{R}$.

Assume that there exist a closed vector subspace $\mathrm{H}^{+}$and a finite-dimensional vector subspace $H^{-}$of $H$ such that $H^{+} \oplus H^{-}$is a direct decomposition of $H$ and

(i) $J(0)=0$ and $D J(0)=0$.

(ii) $\left[D J\left(x+y_{2}\right)-D J\left(x+y_{1}\right)\right]\left[-\left(y_{2}-y_{1}\right)\right]>0 \forall x \in B_{H^{+}}^{\prime}(0, \delta), y_{1}, y_{2} \in$ $B_{H^{-}}^{\prime}(0, \delta)$ and $y_{1} \neq y_{2}$.

(iii) $D J(x+y)(x-y)>0 \quad \forall x \in B_{H^{+}}^{\prime}(0, \delta), y \in B_{H^{-}}^{\prime}(0, \delta)$, and $(x, y) \neq$ $(0,0)$.

(iv) $D J(x) x>p(\|x\|) \quad \forall x \in B_{H^{+}}^{\prime}(0, \delta) \backslash\{0\}$, where $p:(0, \delta] \rightarrow(0, \infty)$ is a non-decreasing function.

Then there exist a positive real number $\epsilon$, an open neighborhood $U$ of 0 in $H$ and an isomorphism $\phi$ from $B_{H^{+}}\left(0, \sqrt{\frac{p(\epsilon / 2)}{2}}\right) \times B_{H^{-}}\left(0, \sqrt{\frac{p(\epsilon / 2)}{2}}\right)$ onto $U$ such that:

$$
J(\phi(x+y))=\|x\|^{2}-\|y\|^{2} \quad \forall(x, y) \in B_{H^{+}}\left(0, \sqrt{\frac{p(\epsilon / 2)}{2}}\right) \times B_{H^{-}}\left(0, \sqrt{\frac{p(\epsilon / 2)}{2}}\right) .
$$

Remark 1.1. Our Morse-Palais lemma is stronger than the classical one even in the case of finite-dimensional normed spaces and is applicable to the following function:

$$
J(x, y)=x^{2}-y^{2}+|x|^{3 / 2} \forall(x, y) \in \mathbb{R}^{2} .
$$

We note that the results in [6, 7] cannot be applied to this case.

Remark 1.2. Let $H^{+}$and $H^{-}$be closed vector subspaces of a normed space $(H,\|\|$. such that the dimension of $H^{-}$is finite and $H^{+} \oplus H^{-}$is a direct decomposition of $H$. Let $J_{1,1}$ and $J_{1,2}$ be the restrictions of continuous bilinear real mappings on $B_{H^{+}}^{\prime}(0,2 \delta)$ and $B_{H^{-}}^{\prime}(0,2 \delta)$ with a positive real number $\delta$ respectively. Assume that there is a positive real number $\eta$ such that

$$
\begin{array}{lc}
J_{1,1}(x, x) \geq \eta\|x\|^{2} & \forall x \in B_{H^{+}}^{\prime}(0,2 \delta) \text { and } \\
J_{1,2}(y, y) \leq-\eta\|y\|^{2} & \forall y \in B_{H^{-}}^{\prime}(0,2 \delta) .
\end{array}
$$

Let $J_{2}$ be a Fréchet differentiable real function on $B_{H^{+}}^{\prime}(0,2 \delta) \times B_{H^{-}}^{\prime}(0,2 \delta)$. Assume there is a positive real number $M$ such that for any $\left(x, y_{1}\right)$ and $\left(x, y_{2}\right)$ in $B_{H^{+}}^{\prime}(0,2 \delta) \times B_{H^{-}}^{\prime}(0,2 \delta)$ we have

$$
\begin{aligned}
& \left\|D J_{2}\left(x, y_{1}\right)\right\| \leq M\left\|\left(x, y_{1}\right)\right\| \text { and } \\
& \left\|D J_{2}\left(x, y_{1}\right)-D J_{2}\left(x, y_{2}\right)\right\| \leq M\left\|y_{1}-y_{2}\right\| .
\end{aligned}
$$

Put $J_{1}(x, y)=J_{1,1}(x, x)+J_{1,2}(y, y)$ for any $(x, y)$ in $B_{H^{+}}^{\prime}(0,2 \delta) \times B_{H^{-}}^{\prime}(0,2 \delta)$ and $J=J_{1}+\epsilon J_{2}$.

Then $J$ satisfies the conditions of Theorem 1.1 for a sufficiently small positive real number $\epsilon$. The $J_{1}$ associated with problem $(P)$ belongs to the class of functionals considered in this remark.

Remark 1.3. In Theorem 1.1 we can replace the conditions (ii) and (iii) by the following:

(v) $\left[D J\left(x_{2}+y_{2}\right)-D J\left(x_{1}+y_{1}\right)\right]\left[\left(x_{2}-x_{1}\right)-\left(y_{2}-y_{1}\right)\right]>0 \forall x_{1}, x_{2} \in B_{H^{+}}^{\prime}(0, \delta) ; y_{1}$, $y_{2} \in B_{H^{-}}^{\prime}(0, \delta)$ and $x_{1}+y_{1} \neq x_{2}+y_{2}$. 
To get the Morse-Palais lemma one uses powerful tools in functional analysis such as the implicit theorem or flows and deformations in Banach spaces. In this paper we only apply elementary differential calculus to get results, hence we do not need the conditions essential for those theories. Therefore our result is stronger than the classical one but our proof is very simple. Of course we cannot get the differentiability of the map $\phi$, but in some problems involving the topological degree we only need the continuity of $\phi$ (see $2,[5,8]$ ). The differentiability of $J$ in our theorem is very weak but sufficient for many applications (see [1, 3, 4]). We shall prove our theorem in the second section.

\section{Proof of the THEOREM}

The proof of the theorem consists of the following lemmas.

Lemma 2.1. There exists a positive real number $\epsilon_{1}<\delta$ having the following property: for each $x \in B_{H^{+}}\left(0, \epsilon_{1}\right)$ there exists a unique $\varphi(x) \in B_{H^{-}}(0, \delta)$ such that $J(x+\varphi(x))=\max \left\{J(x+y): y \in B_{H^{-}}(0, \delta)\right\}$.

Proof. First we show the uniqueness of $\varphi(x)$. Assume by contradiction that there exist $z$ and $z^{\prime}$ in $B_{H^{-}}(0, \delta)$ and $z \neq z^{\prime}$ such that

$$
J(x+z)=J\left(x+z^{\prime}\right)=\max \left\{J(x+y): y \in B_{H^{-}}(0, \delta)\right\} .
$$

This implies $D J(x+z) h=D J\left(x+z^{\prime}\right) h=0$ for any $h$ in $H^{-}$. Choosing $h=z-z^{\prime}$, we get $\left(D J(x+z)-D J\left(x+z^{\prime}\right)\right)\left(z-z^{\prime}\right)=0$, which contradicts (ii).

Now, we prove the existence of $\epsilon_{1}$. Note that $B_{H^{-}}^{\prime}(0, \delta)$ is compact. Assume by contradiction that there exist a sequence $\left\{x_{n}\right\}$ converging to 0 in $B_{H^{+}}(0, \delta)$ and a sequence $\left\{y_{n}\right\}$ in $\partial B_{H^{-}}(0, \delta)$ such that

$$
J\left(x_{n}+y_{n}\right)>J\left(x_{n}+y\right) \quad \forall y \in B_{H^{-}}(0, \delta) .
$$

By the compactness of $\partial B_{H^{-}}(0, \delta)$, we may assume that $\left\{y_{n}\right\}$ converges to $y_{0}$ in $\partial B_{H^{-}}(0, \delta)$. By the continuity of $J$, we have

$$
\lim _{n \rightarrow \infty} J\left(x_{n}+y_{n}\right)=J\left(y_{0}\right) \text { and } \lim _{n \rightarrow \infty} J\left(x_{n}\right)=J(0) .
$$

This implies $J\left(y_{0}\right) \geq J(0)$.

On the other hand, by the mean value theorem and (iii), there exists a real number $t_{y} \in(0,1)$ such that

$$
J\left(y_{0}\right)-J(0)=D J\left(t_{y} \cdot y_{0}\right)\left(y_{0}\right)=-\frac{1}{t_{y}} D J\left(t_{y} \cdot y_{0}\right)\left(-t_{y} \cdot y_{0}\right)<0,
$$

which is a contradiction.

Arguing as above, we may assume further that $\varphi(x) \in B_{H^{-}}(0, \delta / 2)$ for any $x$ in $B_{H^{+}}\left(0, \epsilon_{1}\right)$.

Lemma 2.2. $\varphi$ is continuous on $B_{H^{+}}\left(0, \varepsilon_{1}\right)$.

Proof. Let $\left\{x_{n}\right\}$ be a sequence converging to $x_{0}$ in $B_{H^{+}}\left(0, \epsilon_{1}\right)$. By the compactness of $B_{H^{-}}^{\prime}(0, \delta / 2)$, without loss of generality we may assume that $\left\{\varphi\left(x_{n}\right)\right\}$ converges to $y_{0} \in B_{H^{-}}^{\prime}(0, \delta / 2)$. We have

$$
J\left(x_{n}+\varphi\left(x_{n}\right)\right) \geq J\left(x_{n}+y\right) \quad \forall n \in \mathbb{N}, y \in B_{H^{-}}(0, \delta) .
$$

Taking the limits of both sides of the inequality, we see that $J\left(x_{0}+y_{0}\right) \geq J\left(x_{0}+y\right)$ for every $y$ in $B_{H^{-}}(0, \delta)$. Thus by the uniqueness of $\varphi\left(x_{0}\right), y_{0}$ should be $\varphi\left(x_{0}\right)$, which implies the continuity of $\varphi$. 
Lemma 2.3. Put $j(x)=J(x+\varphi(x))$ for any $x$ in $B_{H^{+}}\left(0, \varepsilon_{1}\right)$. Then $j$ is a continuously directional differentiable real function on $B_{H^{+}}\left(0, \varepsilon_{1}\right)$ and

$$
D j(x) h=D J(x+\varphi(x)) h \quad \forall h \in H^{+}, x \in B\left(0, \varepsilon_{1}\right) .
$$

Proof. Fix an $(x, h)$ in $B_{H^{+}}\left(0, \varepsilon_{1}\right) \times H^{+}$. By the continuously directional differentiability of $J$, the continuity of $\varphi$ and the mean value theorem, there exists $\theta_{t} \in(0,1)$ such that

$$
\begin{aligned}
& \lim _{t \rightarrow 0} \frac{J(x+t h+\varphi(x+t h))-J(x+\varphi(x+t h))}{t} \\
& \quad=\lim _{t \rightarrow 0} D J\left(x+\theta_{t} t h+\varphi(x+t h)\right) h=D J(x+\varphi(x)) h .
\end{aligned}
$$

On the other hand we have

$$
\lim _{t \rightarrow 0} \frac{J(x+t h+\varphi(x))-J(x+\varphi(x))}{t}=D J(x+\varphi(x)) h .
$$

Furthermore, by the maximum property of $\varphi$,

$$
\begin{gathered}
J(x+t h+\varphi(x))-J(x+\varphi(x)) \leq J(x+t h+\varphi(x+t h))-J(x+\varphi(x)) \\
\leq J(x+t h+\varphi(x+t h))-J(x+\varphi(x+t h)) .
\end{gathered}
$$

Combining (2.2), (2.3), and (2.4) we have

$$
\lim _{t \rightarrow 0} \frac{J(x+t h+\varphi(x+t h))-J(x+\varphi(x))}{t}=D J(x+\varphi(x)) h .
$$

Lemma 2.4. We define

$$
\begin{aligned}
& \psi_{1}(x+y)= \begin{cases}\frac{\sqrt{J(x+\varphi(x))}}{\|x\|} x & \text { if } x \neq 0, \\
0 & \text { if } x=0,\end{cases} \\
& \psi_{2}(x+y)= \begin{cases}\frac{\sqrt{J(x+\varphi(x))-J(x+y)}}{\|y-\varphi(x)\|}(y-\varphi(x)) & \text { if } y \neq \varphi(x), \\
0 & \text { if } y=\varphi(x),\end{cases} \\
& \psi(x+y)=\psi_{1}(x+y)+\psi_{2}(x+y) \quad \forall x \in B_{H^{+}}\left(0, \epsilon_{1}\right), y \in B_{H^{-}}(0, \delta) .
\end{aligned}
$$

Then $\psi_{1}, \psi_{2}$, and $\psi$ are continuous on $B_{H^{+}}\left(0, \epsilon_{1}\right) \times B_{H^{-}}(0, \delta)$ and

(2.5) $J(x+y)=\left\|\psi_{1}(x+y)\right\|^{2}-\left\|\psi_{2}(x+y)\right\|^{2} \forall x \in B_{H^{+}}\left(0, \epsilon_{1}\right), y \in B_{H^{-}}(0, \delta)$.

Proof. By the continuity of $J$ and $\varphi$, we see that $\psi_{1}, \psi_{2}$, and $\psi$ are continuous on $B_{H^{+}}\left(0, \epsilon_{1}\right) \times B_{H^{-}}(0, \delta)$. By a straightforward computation we get the last conclusion of the lemma.

Lemma 2.5. $\psi$ is one-to-one on $B_{H^{+}}\left(0, \epsilon_{1}\right) \times B_{H^{-}}(0, \delta)$.

Proof. Assume by contradiction that there exist $x_{1}$ and $x_{2}$ in $B_{H^{+}}\left(0, \epsilon_{1}\right)$ and $y_{1}$ and $y_{2}$ in $B_{H^{-}}(0, \delta)$ such that $x_{1}+y_{1} \neq x_{2}+y_{2}$ and $\psi\left(x_{1}+y_{1}\right)=\psi\left(x_{2}+y_{2}\right)$. By the definition of $\psi$, we have

$$
\begin{aligned}
& \frac{x_{1}}{\left\|x_{1}\right\|}=\frac{x_{2}}{\left\|x_{2}\right\|}, \\
& \frac{y_{1}-\varphi\left(x_{1}\right)}{\left\|y_{1}-\varphi\left(x_{1}\right)\right\|}=\frac{y_{2}-\varphi\left(x_{2}\right)}{\left\|y_{2}-\varphi\left(x_{2}\right)\right\|}, \\
& j\left(x_{1}\right)=J\left(x_{1}+\varphi\left(x_{1}\right)\right)=J\left(x_{2}+\varphi\left(x_{2}\right)\right)=j\left(x_{2}\right), \\
& J\left(x_{1}+y_{1}\right)=J\left(x_{2}+y_{2}\right) .
\end{aligned}
$$


Without loss of any generality, we may assume $x_{2}=s \cdot x_{1}$ with $s \geq 1$. Put $f(t)=$ $j\left(t x_{1}\right)=J\left(t x_{1}+\varphi\left(t x_{1}\right)\right)$ for every $t \in(0, s)$. By Lemma 2.3 and condition (iii), we have

$f^{\prime}(t)=D j\left(t x_{1}\right) x_{1}=D J\left(t x_{1}+\varphi\left(t x_{1}\right)\right) x_{1}=\frac{1}{t} D J\left(t x_{1}+\varphi\left(t x_{1}\right)\right)\left(t x_{1}-\varphi\left(t x_{1}\right)\right)>0$.

Thus $f$ is strictly increasing, $s$ should be equal to 1 and $x_{1}=x_{2}$.

Moreover, we have $J\left(x_{1}+y_{1}\right)=J\left(x_{2}+y_{2}\right)$ and we may suppose that $y_{2}-\varphi\left(x_{2}\right)=$ $y_{2}-\varphi\left(x_{1}\right)=r\left(y_{1}-\varphi\left(x_{1}\right)\right)$ with $r>1$. Thus

$$
\begin{aligned}
J\left(x_{1}+\varphi\left(x_{1}\right)+y_{1}-\varphi\left(x_{1}\right)\right) & =J\left(x_{1}+y_{1}\right)=J\left(x_{2}+y_{2}\right) \\
& =J\left(x_{1}+\varphi\left(x_{1}\right)+r\left(y_{1}-\varphi\left(x_{1}\right)\right)\right) .
\end{aligned}
$$

By the mean value theorem, there exists $t_{x} \in(1, r)$ such that

$$
\begin{aligned}
J\left(x_{1}\right. & \left.+\varphi\left(x_{1}\right)+r\left(y_{1}-\varphi\left(x_{1}\right)\right)\right)-J\left(x_{1}+\varphi\left(x_{1}\right)+y_{1}-\varphi\left(x_{1}\right)\right) \\
& =D J\left(x_{1}+\varphi\left(x_{1}\right)+t_{x}\left(y_{1}-\varphi\left(x_{1}\right)\right)\right)\left((r-1) \cdot\left(y_{1}-\varphi\left(x_{1}\right)\right)\right) \\
& =\frac{r-1}{t_{x}} D J\left(x_{1}+\varphi\left(x_{1}\right)+t_{x}\left(y_{1}-\varphi\left(x_{1}\right)\right)\right)\left(t_{x} \cdot\left(y_{1}-\varphi\left(x_{1}\right)\right)\right) \\
& =\frac{r-1}{t_{x}}\left[D J\left(x_{1}+\varphi\left(x_{1}\right)+t_{x}\left(y_{1}-\varphi\left(x_{1}\right)\right)\right)-D J\left(x_{1}+\varphi\left(x_{1}\right)\right)\right]\left[t_{x}\left(y_{1}-\varphi\left(x_{1}\right)\right)\right] \\
& <0,
\end{aligned}
$$

which is absurd and $\psi$ should be one-to-one on $B_{H^{+}}\left(0, \epsilon_{1}\right) \times B_{H^{-}}(0, \delta)$.

Lemma 2.6. There is a positive real number $\varepsilon<\varepsilon_{0}$ such that

$$
B_{H^{+}}\left(0, \frac{\sqrt{p(\epsilon / 2)}}{\sqrt{2}}\right) \times B_{H^{-}}\left(0, \frac{\sqrt{p(\epsilon / 2)}}{\sqrt{2}}\right) \subset \psi\left(B_{H^{+}}(0, \epsilon) \times B_{H^{-}}(0, \delta)\right) .
$$

Proof. By the mean value theorem, for every $y \in B_{H^{-}}^{\prime}(0, \delta) \backslash\{0\}$ there exists $t_{y} \in(0,1)$ such that

$$
J(y)=J(y)-J(0)=D J\left(t_{y} \cdot y\right) y=\frac{-1}{t_{y}} \cdot D J\left(t_{y} \cdot y\right)\left(-t_{y} \cdot y\right)<0 .
$$

Since $\partial B_{H^{-}}^{\prime}(0, \delta)$ is compact, it implies that there exists a positive real number $C$ such that

$$
J(y)<-C \quad \forall y \in \partial B_{H^{-}}^{\prime}(0, \delta) .
$$

We shall prove that there exists a positive real number $\epsilon \leq \frac{\epsilon_{1}}{2}$ such that

$$
J(x+y) \leq 0 \quad \forall(x, y) \in B_{H^{+}}^{\prime}(0, \epsilon) \times \partial B_{H^{-}}^{\prime}(0, \delta) .
$$

Assume by contradiction that there exists a sequence $\left\{\left(x_{n}, y_{n}\right)\right\}$ in $B_{H^{+}}^{\prime}(0, \epsilon) \times$ $\partial B_{H^{-}}^{\prime}(0, \delta)$ such that $x_{n}$ converges to 0 and $J\left(x_{n}+y_{n}\right)>0$ for any integer $n$.

Because $\partial B_{H^{-}}^{\prime}(0, \delta)$ is compact, we can assume that $\left\{y_{n}\right\}$ converges to $y_{0}$ in $\partial B_{H^{-}}^{\prime}(0, \delta)$. Thus, by the continuity of $J$, we see that $J\left(y_{0}\right) \geq 0$, which contradicts (2.6). Therefore we get (2.7).

We choose $\varepsilon$ in (2.7) such that

$$
\varphi\left(B_{H^{+}}(0, \epsilon)\right) \subset B_{H^{-}}(0, \delta / 2) .
$$


Now, fixing $x$ in $B_{H^{+}}^{\prime}(0, \epsilon)$, by the mean value theorem and condition (iv) there exists $s_{x} \in(1 / 2,1)$ such that

$$
\begin{aligned}
J(x+\varphi(x)) & \geq J(x)>J(x)-J(x / 2)=D J\left(s_{x} x\right)(x / 2) \\
& =\frac{1}{2 s_{x}} D J\left(s_{x} x\right)\left(s_{x} \cdot x\right)>\frac{1}{2} p\left(\left\|s_{x} x\right\|\right) \geq \frac{1}{2} p(\|x / 2\|) .
\end{aligned}
$$

Combining (2.7) and (2.9) we get

$$
\begin{aligned}
J(x+\varphi(x))-J(x+y) \geq J(x+\varphi(x)) \geq & J(x)>\frac{1}{2} p(\|x / 2\|)=\frac{p(\epsilon / 2)}{2} \\
& \forall(x, y) \in \partial B_{H^{+}}^{\prime}(0, \epsilon) \times \partial B_{H^{-}}^{\prime}(0, \delta) .
\end{aligned}
$$

By the continuity of $J$ and the definition of $\psi_{1}$, it follows that

$$
\left\{\frac{t}{\|x\|} x: 0 \leq t \leq \sqrt{\frac{p(\epsilon / 2)}{2}}\right\} \subset \psi_{1}\left(B_{H^{+}}(0, \epsilon) \times B_{H^{-}}(0, \delta)\right) \forall x \in \partial B_{H^{+}}(0, \epsilon) .
$$

Now let $z \in \partial B_{H^{-}}(0, \delta / 2)$ and let $x \in B_{H^{+}}(0, \epsilon)$. By (2.8), there exists $k_{z} \geq 1$ such that $y=k_{z} \cdot z+\varphi(x)$ belongs to $\partial B_{H^{-}}(0, \delta)$. Thus by the continuity of $J,(2.9)$ and the definition of $\psi_{2}$, we have

$$
\left\{\frac{t}{\|z\|} z: 0 \leq t \leq \sqrt{\frac{p(\epsilon / 2)}{2}}\right\} \subset \psi_{2}\left(B_{H^{+}}(0, \epsilon) \times B_{H^{-}}(0, \delta)\right) .
$$

Combining (2.10) and (2.11) proves the lemma.

Lemma 2.7. Put

$$
U=\left(B_{H^{+}}(0, \epsilon) \times B_{H^{-}}(0, \delta)\right) \cap \psi^{-1}\left(B_{H^{+}}\left(0, \frac{\sqrt{p(\epsilon / 2)}}{\sqrt{2}}\right) \times B_{H^{-}}\left(0, \frac{\sqrt{p(\epsilon / 2)}}{\sqrt{2}}\right)\right)
$$

and let $\phi$ be the restriction of $\psi^{-1}$ on $B_{H^{+}}\left(0, \frac{\sqrt{p(\epsilon / 2)}}{\sqrt{2}}\right) \times B_{H^{-}}\left(0, \frac{\sqrt{p(\epsilon / 2)}}{\sqrt{2}}\right)$.

Then

(i) $\phi$ is continuous on $B_{H^{+}}\left(0, \frac{\sqrt{p(\epsilon / 2)}}{\sqrt{2}}\right) \times B_{H^{-}}\left(0, \frac{\sqrt{p(\epsilon / 2)}}{\sqrt{2}}\right)$ and

(ii) $J(\phi(x+y))=\|x\|^{2}-\|y\|^{2} \forall(x, y) \in B_{H^{+}}\left(0, \sqrt{\frac{p(\epsilon / 2)}{2}}\right) \times B_{H^{-}}\left(0, \sqrt{\frac{p(\epsilon / 2)}{2}}\right)$.

Proof. Let $\left(x_{0}, y_{0}\right) \in U$ and let $\left\{\left(x_{n}, y_{n}\right)\right\}$ be a sequence in $U$ such that $\left\{\psi\left(x_{n}, y_{n}\right)\right\}$ converges to $\psi\left(x_{0}, y_{0}\right)$. We prove that $\left\{\left(x_{n}, y_{n}\right)\right\}$ converges to $\left(x_{0}, y_{0}\right)$. By definition we see that $\left\{\psi_{1}\left(x_{n}, y_{n}\right)\right\}$ converges to $\psi_{1}\left(x_{0}, y_{0}\right)$.

We prove that $\left\{x_{n}\right\}$ converges to $x_{0}$. We consider two cases:

Case 1. $x_{0}=0$. We show that $\left\{x_{n}\right\}$ converges to 0 . First note that $\left\{\left\|\psi_{1}\left(x_{n}, y_{n}\right)\right\|\right\}$ converges to 0. Thus, $\left\{j\left(x_{n}\right)\right\}$ converges to 0. Arguing as in Lemma 2.6. we have $j\left(x_{n}\right)>\frac{1}{2} p\left(\left\|x_{n} / 2\right\|\right)$. Therefore $\left\{x_{n}\right\}$ converges to 0 because of the non-decreasing of $p$.

Case 2. $x_{0} \neq 0$. We prove that $\left\{x_{n}\right\}$ converges to $x_{0}$. Note that $\left\{\left\|\psi_{1}\left(x_{n}, y_{n}\right)\right\|\right\}$ converges to $\left\|\psi_{1}\left(x_{0}, y_{0}\right)\right\|$. Thus, $\left\{j\left(x_{n}\right)\right\}$ converges to $j\left(x_{0}\right)>0$ and $\left\{x_{n}\right\}$ does not converge to 0 .

Since $\left\{\frac{\sqrt{j\left(x_{n}\right)}}{\left\|x_{n}\right\|} x_{n}\right\}$ converges to $\frac{\sqrt{j\left(x_{0}\right)}}{\left\|x_{0}\right\|} x_{0}$, it follows that $\left\{\sqrt{j\left(x_{n}\right)}\right\}$ converges to $\sqrt{j\left(x_{0}\right)}$ and then $\left\{\frac{x_{n}}{\left\|x_{n}\right\|}\right\}$ converges to $\frac{x_{0}}{\left\|x_{0}\right\|}$. On the other hand, $\left\|x_{n}\right\|<\delta$ 
for every $n \in \mathbb{N}$. Thus we may assume that $\left\{\left\|x_{n}\right\|\right\}$ converges to $\alpha \in(0, \delta]$. This implies $\left\{x_{n}\right\}$ converges to $\frac{\alpha}{\left\|x_{0}\right\|} x_{0}$.

By the continuity of $j$, we get $\left\{j\left(x_{n}\right)\right\}$ converges to $j\left(\frac{\alpha}{\left\|x_{0}\right\|} x_{0}\right)$, which implies $j\left(x_{0}\right)=j\left(\frac{\alpha}{\left\|x_{0}\right\|} x_{0}\right)$. Since $\psi$ is one-to-one on $U$, we get $\alpha=\left\|x_{0}\right\|$. Therefore $\left\{x_{n}\right\}$ converges to $x_{0}$.

Furthermore, by the compactness of $B_{H^{-}}^{\prime}(0, \delta)$, we can suppose that $\left\{y_{n}\right\}$ converges to $y$ in $B_{H^{-}}^{\prime}(0, \delta)$. By the continuity of $\varphi$ and $J$, we see that $\left\{\varphi\left(x_{n}\right)\right\}$ and $\left\{\sqrt{J\left(x_{n}+\varphi\left(x_{n}\right)\right)-J\left(x_{n}+y_{n}\right)}\right\}$ converge to $\varphi\left(x_{0}\right)$ and $\sqrt{J\left(x_{0}+\varphi\left(x_{0}\right)\right)-J\left(x_{0}+y\right)}$ respectively. Thus we get $\psi_{1}\left(x_{0}, y_{0}\right)=\psi_{1}\left(x_{0}, y\right)$ and $\psi_{2}\left(x_{0}, y_{0}\right)=\psi_{2}\left(x_{0}, y\right)$. Therefore by Lemma 2.5. $\left(x_{0}, y_{0}\right)=\left(x_{0}, y\right)$ and $\phi$ is continuous on $U$.

Using (2.5), we get (i) and (ii).

\section{REFERENCES}

[1] L.H. An, P.X. Du, D.M. Duc, and P.V. Tuoc, Lagrange multipliers for functions derivable along directions in a linear subspace, Proc. Amer. Math. Soc 133 (2005), 595-604. MR:2093084 (2005f:90152)

[2] K.C. Chang, Infinite Dimensional Morse Theory and Multiple Solution Problems, Birkhauser (1993). MR $1196690(94 \mathrm{e}: 58023)$

[3] D.M. Duc, Nonlinear singular elliptic equations, London Math. Soc. 40 (1989), pp. 420-440. MR.1053612 (91g:35107)

[4] D.M. Duc and N.T. Vu, Nonuniformly elliptic equations of $p$-Laplacian type, Nonlinear Analysis, 61 (2005), pp. 1483-1495. MR2135821 (2005k:35111)

[5] H. Hofer, The topological degree at a critical point of mountain-pass type, Proc. Sym. Pure. Math., 45 (1986), Part 1, pp. 501-509. MR0843584 (87h:58031)

[6] N. Kuiper, $C^{1}$-equivalence of functions near isolated critical points, Symposium on infinite dimensional Topology, Ann, Math. Studies 69 Princeton Univ. Press, Princeton (1972), pp. 199-218. MR0413161 (54:1282)

[7] C. Li, S. Li, and J. Liu, Splitting theorem, Poincare-Hopf theorem and Jumping nonlinear problems, Functional Analysis, 221 (2005), pp. 439-455. MR2124871

[8] J. Mawhin and M. Willem, Critical point theory and Hamiltonian systems, Appl. Math. Sci., Springer-Verlag 74 (1989). MR0982267 (90e:58016)

[9] R.S. Palais, Morse theory on Hilbert manifolds, Topology, 2 (1963), pp. 299-340. MR0158410 $(28: 1633)$

Department of Mathematics-Informatics, National University of Hochiminh City, VIETNAM

E-mail address: dmduc@hcmc.netnam.vn

Department of Mathematics-Informatics, National University of Hochiminh City, VIETNAM

E-mail address: vhungt@hcm.fpt.vn

Department of Mathematics-Informatics, National University of Hochiminh City, VIETNAM

E-mail address: Than_Phongnym@yahoo.com 